\title{
Differential outcome of schizophrenia: where we are and where we would like to be ${ }^{\dagger}$
}

Oye Gureje and Alex Cohen

\section{Summary}

Studies examining comparative outcomes of schizophrenia in high-income countries with those in low- and middle-income countries remain of interest to researchers and may be of value in understanding some environmental factors that influence the course and outcome of the disorder. The view that the disorder has a better outcome in low- and middleincome countries compared with high-income countries, even though widespread and supported by a set of World
Health Organization (WHO) studies, requires further testing and exploration. Unfortunately, although not insurmountable, the obstacles for such studies both in terms of implementation and interpretation are considerable.

\section{Declaration of interest}

None.
Oye Gureje (pictured) is President of the African Association of Psychiatrists and Allied Professionals and has conducted clinical studies on schizophrenia and large surveys of mental disorders in primary care settings and in the community. Alex Cohen is an anthropologist with a long-standing interest in cross-cultural research on schizophrenia as well as mental health services in primary care.

The determination of the prognosis of a disorder has implications far beyond the important one of being able to inform patients and their relatives about how their illness may evolve over time. In psychiatry, prognosis has been used as a defining characteristic of some mental disorders. It was central to Kraepelin's idea of dementia praecox ${ }^{1}$ and is still, today, an important feature of how schizophrenia is defined both in the ICD- $10^{2}$ and DSM-IV. ${ }^{3}$ Of course, the more we know about the natural course of a disorder, the more we are able to determine the effectiveness of any treatment we offer to alleviate it.

Contrary to the early views of Kraepelin (which he later questioned) suggesting that schizophrenia is characterised by a poor outcome, studies demonstrating a diversity of outcomes have been conducted over the past 30 years, and these findings have been replicated over and over. Nevertheless, the study of outcome in schizophrenia continues to be of importance partly because the old belief about the uniformly poor prognosis of the disorder is still very much alive in the minds of clinicians around the world. Interest in the study of outcome, especially when comparisons are made between patient groups, also derives from the well-known findings of three World Health Organization (WHO) studies that suggest that the outcome of schizophrenia varied according to the societies in which the individuals lived. ${ }^{4-6}$

\section{WHO studies of course and outcome of schizophrenia}

The WHO International Pilot Study of Schizophrenia (IPSS) was set up to examine the feasibility of making cross-culturally reliable diagnosis of the disorder and to demonstrate the existence of schizophrenia in diverse cultural settings. ${ }^{4}$ In conducting a

†See pp. 194-201, this issue. follow-up on the cohort, the investigators unexpectedly found a markedly better overall outcome in individuals with schizophrenia in India and Nigeria at 2 and 5 years. A subsequent study, designed to be more epidemiologically rigorous and therefore more representative, the Determinants of Outcome of Severe Mental Disorders (DOSMeD), later confirmed these findings. ${ }^{6}$ High rates of complete clinical remission were significantly more common in low- and middle-income countries (37\%) than in high-income countries (15.5\%). Also, even though individuals in low- and middle-income countries were much less likely to be on continuous antipsychotic medication, they nevertheless experienced significantly longer periods of unimpaired functioning when compared with individuals from the high-income country study sites. Even then, the findings were more indicative of a diversity of outcomes rather than any culture-specific pattern of outcome. For example, there was no significant difference in the proportions of continuous unremitting illness (11.1 and 17.4\%) across the two types of settings. The International Study of Schizophrenia, ${ }^{5}$ which was a long-term follow-up of participants in IPSS and DOSMeD, supported the earlier findings.

Irrespective of the findings reported by these studies, it is clear that most commentators have not given their findings the nuanced interpretation that the results deserve. Thus, it is true that it has almost become axiomatic to claim that schizophrenia has a better course and outcome in low- and middle-income countries. ${ }^{7}$ The situation has not been helped by the lack of studies of comparable rigor and coverage that would provide convincing refutation or replication of the main findings of the WHO studies. Nevertheless, evidence from several other studies, albeit of diverse coverage and rigor, suggests that the strikingly better outcome reported for a substantial proportion of participants from lowand middle-income country sites in the WHO studies may have been atypical of people with schizophrenia from those countries. ${ }^{7}$

\section{The importance of differential outcome}

The suggestion that schizophrenia might have a better outcome in settings grouped together on the basis of comparable social factors provides a compelling research question. This is so because, other than biological and individual factors such as genetic vulnerability, the influence of which remains indeterminate, environmental, including sociocultural, factors are of particular importance in 
disentangling prognostic features. Of course, subsumed under sociocultural factors are a great many variables of different complexities both in their assessment and in their interpretation. ${ }^{8}$

More studies examining differential outcomes in diverse sociocultural settings are therefore needed, and the study by Haro et al in this issue of the Journal is an important addition to the literature. ${ }^{9}$ Its strengths include a large sample, serial assessments that utilised standard operational definitions of outcomes and wide geographic diversity. Nevertheless, we think they have allowed the interpretation of their findings to be influenced by those of the 'axiomatic' WHO studies. Thus, even though they state that their ' . . . findings support the earlier WHO studies reporting differences in outcomes between regions,' 9 a closer look at the methodology of the study suggests that the results deserve a more tentative interpretation. First, it is largely a study of prevalent cases that were initiating new antipsychotic treatment or requiring a change in medication (with only $9 \%$ being never treated). Second, the samples varied in the mean duration of illness from 8 to 12 years and in the proportions with a history of substance misuse from 3 to $12 \%$. However, in order to truly provide answers germane to the determination of differential outcome of schizophrenia across settings, studies need to be of incident cases, preferably defined as first episode and untreated. Anything less rigorous invites selection bias, especially when samples are derived from treated populations given that factors relating to access to services and pathways to care vary enormously across settings and, thus, introduce varying levels of chronicity and comorbidity. To be fair, the authors have attempted to account for this in the statistical approach they have employed but this is difficult given that there is little evidence that selection bias is the same in all settings.

Haro et al also make the observation that although North Africa/Middle East had a similar clinical outcome profile with Latin America, the two regions were widely divergent with regard to functional outcome. The same pattern of inconsistent outcome profiles is seen when Northern Europe is compared with Southern Europe, even though both belong to the 'high-income' countries grouping. Clearly, if a neat interpretation of similar outcomes according to developmental stage was applicable, it would be plausible to expect functional outcomes to be more similar within countries with comparable sociocultural and economic status. Of course, even though clinical and functional outcomes can be expected to be unidirectional, one should not dismiss the possibility of discordance given the finding by Strauss \& Carpenter of 'open-linked' systems of outcomes. ${ }^{10}$ That aside, functional remission is a complex multidimensional concept to assess. Its measurement is made even more difficult when trying to compare it across diverse settings. ${ }^{11}$ Therefore, one must wonder whether the definition of functional remission used in this study meant the same thing in the different settings, especially when it does not appear that the cross-cultural validity and reliability of this definition has been determined.

\section{Conducting comparative outcome studies of schizophrenia}

The challenges of conducting ecological epidemiological studies that will throw light on differential outcomes of schizophrenia, although not insurmountable, are nevertheless considerable. ${ }^{12}$ One of the challenges relates to definitional issues, especially when variables are capturing factors of divergent social or cultural import. Features such as occupational/vocational status and extent and nature of social interactions are problematic to define in ways that do sufficient justice to cultural and social diversity while still making it possible to carry out cross-site comparisons. Furthermore, it may not be valid to make cross-cultural comparisons of rates of marriage among people with schizophrenia because the institution of marriage is shaped by social and cultural forces that exert far more influence than psychiatric diagnosis. As demonstrated elsewhere, ${ }^{7}$ it is better to compare marriage rates of people with schizophrenia to the rates found among the general populations in which they live. When this is done, it becomes clear that individuals with schizophrenia have rates of marriage that are relatively low and rates of separation and divorce that are relatively high. Information on mortality, another important outcome variable, does not provide clarity about differential outcome. A recent systematic review of mortality in schizophrenia found no significant difference in standardised mortality ratios between sites grouped according to their economic status. ${ }^{13}$

Interpreting findings on the comparative outcomes of schizophrenia in clinical samples drawn from high-income countries with those from low- and middle-income countries is made more difficult by findings suggesting that a high proportion of people with psychosis in the community in low- and middleincome countries may not have received any formal treatment. ${ }^{14}$ Thus, those who do may represent an atypical group. In addition, the forced dichotomy of 'high-income' and 'low- and middleincome' settings tends to ignore the diverse social, cultural and economic factors embedded within those terms. Although the dichotomy may provide a useful way of exploring a large dataset, it is important not to stretch the implied uniformity between and within the countries so grouped beyond the very rudimentary.

Studies examining the differential outcome of schizophrenia in diverse settings with clearly defined and measurable characteristics are of potential importance in understanding sociocultural factors that may be relevant to the course of the disorder. Such studies need not be limited to between-nation comparisons, but could usefully examine within-nation differences in outcome as well since it is plausible to expect that factors such as urbanicity, migrant status and neighbourhoodlevel variables related to social capital may influence course and outcome. $^{15}$

Oye Gureje, DSC, FRCPsych, WHO Collaborating Center for Research and Training in Mental Health, Neurosciences, Drug and Alcohol Abuse, Department of Psychiatry, University of Ibadan, Nigeria, Africa; Alex Cohen, PhD, Faculty of Epidemiology and Population Health, London School of Hygiene and Tropical Medicine, London, UK

Correspondence: Oye Gureje, WHO Collaborating Center for Research and Training in Mental Health, Neurosciences, Drug and Alcohol Abuse, Department of Psychiatry, University of Ibadan, Nigeria, Africa. Email: ogureje@comui.edu.ng

First received 17 Feb 2011, accepted 28 Mar 2011

\section{References}

1 Kraepelin E. Psychiatrie. 5 Auflage. Barth, 1986.

2 American Psychiatric Association. Diagnostic and Statistical Manual of Mental Disorders (4th edn) (DSM-IV). APA, 1994.

3 World Health Organization. The ICD-10 Classification of Mental and Behavioural Disorders: Clinical Descriptions and Diagnostic Guidelines. WHO, 1992.

4 World Health Organization. Schizophrenia: An International Follow-up Study. John Wiley \& Sons, 1979

5 Hopper K, Harrison G, Janca A, Sartorius N. Recovery from Schizophrenia: An International Perspective. Oxford University Press, 2007.

6 Jablensky A, Sartorius N, Ernberg G, Anker M, Korten A, Cooper JE. Schizophrenia: manifestations, incidence and course in different cultures. A World Health Organization ten-country study. Psychol Med Monogr Supp 1992; 20: 1-97 
7 Cohen A, Patel V, Thara R, Gureje O. Questioning an axiom: better prognosis for schizophrenia in the developing world? Schizophr Bull 2008; 34: 229-44.

8 Edgerton RB, Cohen A. Culture and schizophrenia: the DOSMD challenge. Br J Psychiatry 1994; 164: 222-31.

9 Haro JM, Novick D, Bertsch J, Karagianis J, Dossenbach M, Jones, PB. Cross-national clinical and functional remission rates in the World Schizophrenia Outpatient Health Outcomes (W-SOHO) study. Br J Psychiatry 2011; 199: 194-201.

10 Strauss JS, Carpenter Jr WT. Prediction of outcome in schizophrenia: III. Five-year outcome and its predictors. Arch Gen Psychiatry 1977; 34: 159-63.

11 Isaac $M$, Chand $P$, Murthy $P$. Schizophrenia outcome measures in the wider international community. Br J Psychiatry 2007; 191 (suppl 50): s71-7.
12 Andreasen NC, Carpenter Jr WT, Kane JM, Lasser RA, Marder SR, Weinberger DR. Remission in schizophrenia: proposed criteria and rationale for consensus. Am J Psychiatry 2005; 162: 441-9.

13 Saha S, Chant D, McGrath J. A systematic review of mortality in schizophrenia: is the differential mortality gap worsening over time? Arch Gen Psychiatry 2007; 64: 1123-31.

14 Gureje O, Olowosegun O, Adebayo K, Stein DJ. The prevalence and profile of non-affective psychosis in the Nigerian Survey of Mental Health and Wellbeing. World Psychiatry 2010; 9: 50-5.

15 McGrath JJ. The surprisingly rich contours of schizophrenia epidemiology Arch Gen Psychiatry 2007; 64: 14-6.

\section{Poems \\ by doctors}

\section{Nightwatch, 1842}

\section{Tom Pow}

When night falls and all others have resigned their trust, I walk the galleries, the guardian, the master of all that stalks their fitful sleep. I inquire into all compalints, gratify all reasonable desires. I compel those with angry and turbulent passions to follow healthier trains of thought. I give due ballast to the most frivolous claims become master of reason when I've need to flatter the restless and the noisy: "What spikes your night are pictures," I tell them. One's convinced that shadows cut her like knives, another dreams she's beset by gangs of wizards and thieves. To those who sing or whistle or laugh; or to one who struts the long gallery and chants, "Dirty slut, dirty slut, slut..." I'll bring the required balm. The somnambulist I'Il lead back to bed like a child: while to her who cries for "Auld Auntie Peggy", my soft step nears like a loved one, giving fresh hope and healing to her troubled mind. No sound soils the night that can't be traced back to its primary source. from their soliloquies, songs and prayers, I chart the course of that wayward black river whose stream's one moment chocked by rock and, at the next, split in shallows featureless as smoke. In the solitude of midnight I notate such fractured plots. When day commands the gallery, another will take my watch.

From Dear Alice - Narratives of Madness (Salt, 2008). We have also published two other poems by Tom Pow, The Great Asylums of Scotland and The Last Vision of Angus McKay. Reproduced with permission from Salt Publishing Limited. (C) Tom Pow.

Chosen by Femi Oyebode. 